\title{
Hospital readmission performance and patterns of readmission: retrospective cohort study of Medicare admissions
}

\begin{abstract}
Kumar Dharmarajan cardiology fellow ${ }^{1}$, Angela F Hsieh statistician ${ }^{2}$, Zhenqiu Lin statistician ${ }^{2}$, Héctor Bueno associate professor of medicine ${ }^{3}$, Joseph S Ross assistant professor of medicine ${ }^{4}$, Leora I Horwitz assistant professor of medicine ${ }^{4}$, José Augusto Barreto-Filho associate professor of cardiology ${ }^{5}$, Nancy Kim assistant professor of medicine ${ }^{4}$, Lisa G Suter assistant professor of medicine $^{6}$, Susannah M Bernheim acting director of quality measurement programs ${ }^{2}$, Elizabeth E Drye research scientist in pediatrics ${ }^{2}$, Harlan M Krumholz Harold J Hines Jr professor of medicine ${ }^{2}$
\end{abstract}

'Division of Cardiology, Department of Internal Medicine, Columbia University Medical Center, 630 West 168th Street, Box 93, PH 10-203, New York, NY 10032, USA; ${ }^{2}$ Center for Outcomes Research and Evaluation, Yale-New Haven Hospital, 1 Church Street, Suite 200, New Haven, CT 06510, USA; ${ }^{3}$ Department of Cardiology, Hospital General Universitario Gregorio Marañón, Dr Esquerdo 46 Madrid 28007, Spain; ${ }^{4}$ Section of General Internal Medicine, Yale University School of Medicine, PO Box 208056, New Haven, CT 06520, USA ; ${ }^{5}$ Division of Cardiology, Federal University of Sergipe, and Clínica e Hospital São Lucas, Rua Claudio Batista, S/N, Bairro Santo Antonio, 49060-100, Aracaju, Sergipe, Brazil; ${ }^{6}$ Section of Rheumatology, Yale University School of Medicine, PO Box 208031, New Haven, CT 06520, USA

\begin{abstract}
Objectives To determine whether high performing hospitals with low 30 day risk standardized readmission rates have a lower proportion of readmissions from specific diagnoses and time periods after admission or instead have a similar distribution of readmission diagnoses and timing to lower performing institutions.

Design Retrospective cohort study.

Setting Medicare beneficiaries in the United States.

Participants Patients aged 65 and older who were readmitted within 30 days after hospital admission for heart failure, acute myocardial infarction, or pneumonia in 2007-09.

Main outcome measures Readmission diagnoses were classified with a modified version of the Centers for Medicare and Medicaid Services' condition categories, and readmission timing was classified by day (0-30) after hospital discharge. Hospital 30 day risk standardized readmission rates over the three years of study were calculated with public reporting methods of the US federal government, and hospitals were categorized with bootstrap analysis as having high, average, or low readmission performance for each index condition. High and low performing hospitals had $\geq 95 \%$ probability of having an interval estimate respectively less than or greater than the national 30 day readmission rate over the three
\end{abstract}

year period of study. All remaining hospitals were considered average performers.

Results For readmissions in the 30 days after the index admission, there were 320003 after 1291211 admissions for heart failure (4041 hospitals), 102536 after 517827 admissions for acute myocardial infarction (2378 hospitals), and 208438 after 1135932 admissions for pneumonia (4283 hospitals). The distribution of readmissions by diagnosis was similar across categories of hospital performance for all three conditions. High performing hospitals had fewer readmissions for all common diagnoses. Median time to readmission was similar by hospital performance for heart failure and acute myocardial infarction, though was 1.4 days longer among high versus low performing hospitals for pneumonia $(P<0.001)$. Findings were unchanged after adjustment for other hospital characteristics potentially associated with readmission patterns.

Conclusions High performing hospitals have proportionately fewer 30 day readmissions without differences in readmission diagnoses and timing, suggesting the possible benefit of strategies that lower risk of readmission globally rather than for specific diagnoses or time periods after hospital stay. 


\section{Introduction}

Rates of readmission in the 30 days after an index admissions for heart failure, acute myocardial infarction, or pneumonia remain high and variable across hospitals in the United States. ${ }^{1-3}$ In 2007-09, publicly reported 30 day rates of readmission standardized for risk ranged from $17 \%$ to $33 \%$ for patients admitted with heart failure, $15 \%$ to $27 \%$ for patients admitted with acute myocardial infarction, and $14 \%$ to $26 \%$ for patients admitted with pneumonia. ${ }^{45}$

Little is known about the relation between hospital rates of readmission and the diagnoses and timing of readmissions. For example, do high performing hospitals with the lowest 30 day readmission rates have fewer readmissions due to the same conditions that prompted the initial admission or that occur soon after discharge? If so, readmissions for recurrent illnesses should be less common and the median time to readmission longer at these institutions. In contrast, do high performing hospitals have broadly reduced risk of readmission for the spectrum of conditions necessitating readmission and is this risk of readmission lower for the entire month after discharge? In this case, both the distribution of readmission diagnoses and the median time to readmission should be similar to those of lower performing hospitals even though the absolute number of readmissions is less. Given uncertainties about which strategies are most effective in reducing readmissions in the context of contemporary practice, answers to these questions might illuminate how readmissions have been empirically reduced at hospitals with the lowest rates.

As previous qualitative research has found that hospitals with the lowest 30 day mortality for acute myocardial infarction possess organizational capacities with diffuse salutary impact beyond disease specific processes and protocols, ${ }^{67}$ we hypothesized that high performing hospitals with the lowest 30 day readmission rates would have proportionately fewer readmissions across all diagnoses and time periods after discharge. To test this, we studied a national cohort of beneficiaries of Medicare fee for service health insurance who were readmitted within 30 days after admission for heart failure, acute myocardial infarction, or pneumonia in 2007-09. We examined whether the spectrum of readmission diagnoses and median time to readmission varied by categorization of performance and, if so, whether they varied for better and worse performers in a consistent manner for all three conditions. We focused on patients who were initially admitted for heart failure, acute myocardial infarction, or pneumonia as these illnesses are among the most common causes of admission in older people ${ }^{8}$ and are the focus of performance based reimbursement by the Centers for Medicare and Medicaid Services. ${ }^{4-9}$

\section{Methods}

\section{Data sources and study population}

We used Medicare standard analytic and denominator files to identify index admissions to acute care hospitals in 2007-09 with a principal discharge diagnosis of heart failure, acute myocardial infarction, or pneumonia. Cohorts were defined with ICD-9 (international classification of diseases, 9th revision, clinical modification) codes used in readmission measures publically reported by the Centers for Medicare and Medicaid Services (table A in appendix 1). ${ }^{10-12}$ We included patients aged 65 and older with a complete claims history for one year before admission. Reasons for exclusion included death in hospital, less than 30 days after discharge with enrollment in Medicare fee for service, transfer to another acute care facility, and discharge against medical advice. ${ }^{10-12}$ To provide more reliable estimates of hospital performance, we did not include patients from hospitals with fewer than 25 index admissions for each condition or no readmissions over the study period. We required that hospitals be represented in 2008 American Hospital Association survey data.

We then used definitions consistent with current Centers for Medicare and Medicaid Services measures (table B in appendix 1) to identify all qualifying readmissions for any cause to any short term acute care hospital within 30 days of the index admission. ${ }^{10-12}$ We specifically excluded planned readmissions for revascularization procedures after admission for acute myocardial infarction. As with the Centers for Medicare and Medicaid Services measures, only the first readmission within 30 days of discharge was considered as a 30 day readmission. Additional readmissions within this 30 day period were not counted as 30 day readmissions or index admissions for the same condition. Subsequent admissions occurring after 30 days from discharge were counted as index admissions if they met inclusion criteria. All study analyses were performed on these readmission cohorts.

\section{Categorization of readmission diagnoses and timing}

To characterize readmission patterns across hospitals, we classified individual diagnoses for readmitted patients using a modified version of the Centers for Medicare and Medicaid Services condition categories, as previously described. ${ }^{13}{ }^{14}$ Each of the 189 condition categories is structured around a reasonably well specified disease or medical condition. ${ }^{13}$ Because nearly $90 \%$ of the 189 condition categories each accounted for less than $1 \%$ of all readmissions, however, we consolidated related diagnoses in a shorter list of 30 modified condition categories to make data presentation more clinically meaningful (table $\mathrm{C}$ in appendix 1). Based on our opinion, these 30 modified condition categories were designed to be clinically internally consistent and capture the most common diagnostic categories associated with readmission. Cardiopulmonary diagnoses were subdivided into a larger number of diagnostic categories given their expected importance after index admission for heart failure, acute myocardial infarction, or pneumonia.

We classified timing of readmission by day (0-30) after hospital discharge.

\section{Hospital performance}

For each hospital, we calculated separate 30 day readmission rates standardized for risk after index admission for heart failure, acute myocardial infarction, and pneumonia. The National Quality Forum ${ }^{15}$ approved these measures and an independent committee of statisticians nominated by the Committee of Presidents of the Statistical Societies endorsed the validity of the methods. ${ }^{16}$ Risk standardized readmission rates are publically reported by the Centers for Medicare and Medicaid Services ${ }^{4-9}$ and have been incorporated into incentive programs within the Affordable Care Act. ${ }^{9}$ The modeling strategy used for risk standardization accounts for correlation of observed readmission rates within a hospital and reflects the assumption that, after adjustment for sampling variability and patient characteristics including age, sex, and comorbidities, the remaining variation in readmission rates reflects hospital quality. ${ }^{10-12}$

To compare readmission diagnoses and timing across hospitals of different performance levels, we used the bootstrap algorithm to construct a $95 \%$ interval estimate for each 30 day risk standardized readmission rate and divided hospitals into high, 
average, and low performers for each index condition. ${ }^{10-12}$ High and low performing hospitals had a $95 \%$ or greater probability of having an interval estimate respectively less than or greater than the national rate over the three year period of study. All remaining hospitals were considered average. For each hospital, we calculated performance separately for heart failure, acute myocardial infarction, and pneumonia cohorts.

\section{Outcomes}

\section{Readmission diagnoses by hospital performance}

We identified the percentage of readmissions for each of the 10 most common diagnostic categories by modified condition categories among hospitals with high, average, and low performance and compared these percentages across hospital performance groups.

To deal with potential confounding of results by differences in hospital characteristics among performance groups we also examined whether hospital 30 day risk standardized readmission rates were associated with the percentage of readmissions for each of the 10 most common diagnostic categories by modified condition category after adjustment for hospital factors used in performance reports by the Centers for Medicare and Medicaid Services $^{4}$ and previous studies examining hospital readmissions. ${ }^{17-19}$

\section{Readmission timing by hospital performance}

We calculated the median time to readmission among hospitals with high, average, and low performance and compared this timing across performance categories.

To deal with potential confounding, we also examined whether hospital 30 day risk standardized readmission rates were associated with median time to readmission after adjustment for the additional hospital factors cited above.

\section{Statistical analyses}

\section{Readmission diagnoses by hospital performance}

We first identified the percentage of observed 30 day readmissions for the 30 most common readmission diagnostic categories by modified condition category for each hospital in the heart failure, acute myocardial infarction, and pneumonia cohorts. We also noted each hospital's percentage of observed 30 day readmissions for cardiovascular diagnoses after admissions for heart failure and acute myocardial infarction, and pulmonary diagnoses after admissions for pneumonia. The modified condition category groups comprising cardiovascular and pulmonary diseases are listed in tables $\mathrm{D}$ and $\mathrm{E}$ in appendix 1.

We then calculated a weighted average of the percentage of hospitals' readmissions for the 10 most common readmission diagnoses by modified condition category for high, average, and low performing hospitals. Weighting was proportionate to the number of readmissions for each hospital during 2007-09. We calculated summary statistics for each hospital performance level.

To examine the association between hospital performance and readmission diagnoses across the range of hospital 30 day risk standardized readmission rates, we developed weighted regression models of the relation between the rates and each of the 10 most common readmission diagnoses across hospitals. Regression models were weighted by the inverse of the standard error of the hospital level percentage of each of the 10 most common readmission diagnoses. After univariate analysis, we fitted additional weighted regression models that were adjusted for other hospital characteristics besides the 30 day risk standardized readmission rate including teaching status, urban/rural location, ownership status (private, not for profit, public), safety net status, critical access status, proportion of patients receiving Medicaid health insurance, proportion of African-American patients, and hospital volume with regard to the number of index admissions for each condition. Safety net hospitals in the US provide care for a high proportion of uninsured, underinsured, or Medicaid patients; as has been done previously, we defined safety net hospitals as public or private hospitals with an annual Medicaid caseload >1 SD above their respective state's mean private hospital Medicaid caseload. ${ }^{2021}$

\section{Readmission timing by hospital performance}

We calculated a weighted average of hospitals' median time to readmission for hospitals with high, average, and low performance by 30 day risk standardized readmission rate. We calculated summary statistics for each hospital performance level. We used the Kruskal-Wallis test to examine whether median time to readmission differed between hospital performance groups. We also developed regression models of the relation between hospital level risk standardized readmission rates and hospital level median time to readmission. Models were weighted by the number of readmissions for each hospital. After univariate analysis, we fitted additional weighted regression models that were adjusted for the other hospital characteristics described above.

All significance levels for the regression models were two sided with a $P$ value $<0.05$, and analyses were carried out with SAS 9.2 (SAS Institute, Cary, NC).

\section{Results}

In the 30 days after the index admission, we identified 320003 readmissions after 1291211 index admissions for heart failure (4041 hospitals), 102536 readmissions after 517827 index admissions for acute myocardial infarction (2378 hospitals), and 208438 readmissions after 1135932 index admissions for pneumonia (4283 hospitals). Appendix 2 provides flow diagrams that identify hospital exclusions for each cohort. The average 30 day risk standardized readmission rates were $24.7 \%, 19.8 \%$, and $18.2 \%$ after index admissions for heart failure, acute myocardial infarction, or pneumonia, respectively (table $1 \Downarrow$ ). Hospitals in the acute myocardial infarction cohort were the least likely to be designated as rural or critical access. Compared with high performing hospitals, low performers for heart failure, acute myocardial infarction, and pneumonia had a higher percentage of African-American patients.

\section{Readmission diagnoses by hospital performance}

The percentage of readmissions for the 10 most common diagnostic categories was similar across hospital performance groups for heart failure (fig $1 \Downarrow$ ), acute myocardial infarction (fig $2 \Downarrow$ ), and pneumonia (fig $3 \Downarrow$ ). For high, average, and low performing hospitals, the percentage of readmissions for cardiovascular disease after index admission for heart failure was $53.0 \%, 52.7 \%$, and $53.5 \%$, respectively, while that for acute myocardial infarction was $55.1 \%, 53.6 \%$, and $51.0 \%$, respectively. The percentage of readmissions for pulmonary disease after index admission for pneumonia was 39.2\%, 38.6\%, and $37.0 \%$ for high, average, and low performing hospitals, respectively. 
We found similarly small relations between hospital performance across the range of 30 day risk standardized readmission rates and readmission diagnoses. Univariate analyses (table F in appendix 1) showed that for every standard deviation increase in rate, readmissions for common diagnostic categories changed between $0 \%$ and $0.6 \%$. These associations were typically further reduced in multivariate analyses (table $\mathrm{G}$ in appendix 1). Rural and critical access hospitals were found to have a unique spectrum of readmissions. For example, after admission for acute myocardial infarction, rural and critical access hospitals readmitted $6.6 \%$ and $9.6 \%$, respectively, more patients for recurrent acute myocardial infarction. Readmission for recurrent pneumonia was also more common at these institutions.

\section{Readmission timing by hospital performance}

There were no notable differences in the weighted median time to readmission among high, average, and low performing hospitals for heart failure (12.6, 12.3, and 12.1 days, respectively; $\mathrm{P}=0.11)$ or acute myocardial infarction $(9.8,10.1$, and 10.0 days, respectively; $\mathrm{P}=0.12$ ). High performing hospitals for pneumonia had longer weighted median times to readmission by 1.4 days compared with low performing hospitals (12.9 days $v 11.5$ days, respectively; $\mathrm{P}<0.001$ ) (figs $4 \Downarrow, 5 \Downarrow$, and $6 \Downarrow$, respectively).

We found similar relations between hospital performance across the range of 30 day risk standardized readmission rates and median time to readmission. Univariate analysis showed that for every standard deviation increase in rate, the median time to readmission changed by $0.13,0.04$, and 0.23 days after admission for heart failure, acute myocardial infarction, or pneumonia, respectively. Associations were similar in multivariate analyses (table $2 \Downarrow$ ). All other hospital characteristics were associated with changes in median time to readmission by $\leq 0.45$ days

\section{Discussion}

\section{Principal findings}

In contemporary practice, hospitals with different 30 day readmission rates after index admissions for heart failure, acute myocardial infarction, or pneumonia have a similar distribution of readmissions with regard to their diagnoses and timing. Our national study examined readmission diagnoses and timing patterns across hospitals with different performance profiles. The findings extend previous work on the predictors of hospital performance $\mathrm{e}^{17-19}$ by showing that high performing hospitals with low 30 day risk standardized readmission rates maintain a similar pattern of readmission diagnoses and timing as lower performing institutions. High performers have not empirically achieved low overall rates of readmission by reducing readmissions from specific diagnoses or time periods after discharge. As readmissions result from a diverse spectrum of conditions and occur throughout the month after admission, ${ }^{14-2.5}$ our results suggest that hospitals might best achieve low 30 day readmission rates by using general strategies or capacities that apply broadly across potential readmission diagnoses and time periods after discharge.

\section{Extension of previous research}

We have extended previous research describing the diverse spectrum of readmission diagnoses ${ }^{14-25}$ by showing that medical conditions that cause readmission remain similar across hospitals with different characteristics. For example, in multivariate analysis we found that the percentage of readmissions for each of the 10 most common readmission diagnoses in heart failure, acute myocardial infarction, and pneumonia cohorts varied by less than $1 \%$ at safety net hospitals compared with non-safety net hospitals. We additionally found that every standard deviation increase in hospitals' percentage of patients from ethnic minority groups and patients with Medicaid health insurance resulted in similarly small changes in the percentage of readmissions for common readmission diagnoses. These results show that hospitals serving minority groups have similar readmission patterns, despite having higher overall rates of readmission. ${ }^{26}$ Strategies to reduce readmission could therefore account for a similar underlying spectrum of readmission diagnoses across many different care settings.

\section{Implications for reducing readmissions}

Our findings could explain why the combination of broad based ${ }^{27}$ and longitudinal strategies applicable to a range of potential readmission diagnoses throughout the period after discharge have, in intervention studies, shown efficacy in lowering hospital readmissions while disease specific or time limited interventions have been often unsuccessful. Rich and colleagues showed that complementary interventions delivered by a nurse, dietician, social worker, and geriatrician in the hospital as well as longitudinal follow-up with home care and study teams reduced readmissions after hospital admission for heart failure. ${ }^{28}$ Similarly, Coleman and colleagues found that the combination of inpatient visits and visits after discharge by a nurse transition coach, assistance with self management of drug treatment, education about potential "red flag" conditions that require expedited follow-up, and the creation of a patient owned medical record designed to bridge care settings lowered readmission in patients with 11 common conditions. ${ }^{29}$

Our findings could also explain why disease specific or time limited interventions have generally been found to be less efficacious, as the highest performing hospitals were not especially good at reducing readmissions for particular medical conditions or time periods after discharge. The wide breadth of diagnoses and time periods associated with readmission ${ }^{14-25}$ implies that overall rates of readmission cannot be reduced by lowering only a small subset of readmissions. It is therefore not surprising that major trials of disease specific or singular interventions after discharge have had disappointing results for all cause readmission. ${ }^{30-32}$ In particular, well designed trials of telemonitoring to identify early evidence of heart failure decompensation have not shown reduced rates of all cause readmission, despite involving highly engaged patients and healthcare providers. ${ }^{30} 32$

Our results are also consistent with those of previous studies linking high hospital performance with organizational and cultural characteristics that would be expected to apply broadly to a range of conditions and times after hospital admission. Curry and colleagues conducted a qualitative analysis to identify potential drivers of low risk standardized mortality rates for acute myocardial infarction ${ }^{6}$ and found that high and low performing hospitals were not differentiated by disease specific protocols or processes of care but rather by shared organizational values of providing high quality care, senior managerial provision of financial and non-financial resources, multidisciplinary teams with empowered non-physician providers, strong internal communication and coordination, and commitment to problem solving. The presence of organizational characteristics with the potential to exert broad influence across readmission diagnoses and time periods after discharge among 
high performing hospitals was confirmed in a cross sectional survey of more than 500 hospitals. $^{7}$

The reasons underlying the greater percentage of readmissions for recurrent acute myocardial infarction and recurrent pneumonia at rural and critical access hospitals are unknown. As these hospitals are less likely to have catheterization facilities, ${ }^{33}$ patients treated there for acute myocardial infarction might be less likely to undergo revascularization and more likely to experience reinfarction. Rural hospitals might also less often follow acute myocardial infarction process measures. ${ }^{34-36}$ The reasons for greater recurrence of pneumonia at rural hospitals are less clear but could relate to worse performance on evidence based care processes,$^{33}$ differences in use of resources after acute care, ${ }^{37}$ or greater difficulties accessing care. ${ }^{38} 39$ Despite these uncertainties, the different composition of readmission diagnoses at these facilities could be useful in guiding disease surveillance after hospital discharge.

\section{Social and hospital factors in readmission}

Our finding of similar patterns of readmission regardless of hospital readmission performance could imply deficiencies in the Centers for Medicare and Medicaid Services algorithms we used to calculate risk standardized readmission rates. Differences in hospital performance could fundamentally reflect differences in social and environmental factors experienced by patients at high and low performing hospitals rather than intrinsic signals of hospital quality. There is, however, controversy about the importance of social and environmental factors at the hospital level. The predictive power of these factors at the patient level has been inconsistent. ${ }^{17-46}$ Even clinical factors that describe illness severity at the time of admission, such as vital signs and results of laboratory testing, have not been shown to provide substantial incremental value in predicting rates of hospital readmission compared with administrative claims data. ${ }^{10-47}$

In contrast, there is evidence that hospitals and hospital organizational characteristics can impact rates of readmission. For example, between 1997 and 2010 Veterans Affairs hospitals significantly lowered 30 day readmission rates for heart failure, acute myocardial infarction, pneumonia, and other common conditions ${ }^{48}$ Moreover, hospitals that care for older adults lowered 30 day readmission rates in the last quarter of 2012 in the face of new financial penalties designed to reduce readmission. ${ }^{49}$ In addition, many hospitals with a high percentage of medically underserved patients from either racial and ethnic minority groups or low socioeconomic strata nonetheless have low risk standardized readmission rates. ${ }^{4}$ High performing hospitals might possess particular organizational characteristics and capacities that are responsible for their low rates of adverse outcomes $^{6}$ and are consistent with organizational performance goals for the highest performing healthcare organizations..$^{50}$

\section{Study limitations}

There are potential limitations to this analysis. We restricted our study to beneficiaries of Medicare fee for service health insurance at hospitals with more than 25 index admissions during the study period, so conclusions drawn from this population might not apply to others such as younger patients, patients admitted with different conditions, or patients at the smallest hospitals. We also did not include information from hospitals with no readmissions during the study period, which included 708 hospitals (15.9\% of all hospitals) treating acute myocardial infarction and 313 hospitals (6.5\% of all hospitals) treating pneumonia. Most of these hospitals had small case volumes as the median number of admissions for acute myocardial infarction and pneumonia at these institutions was three and 30 , respectively. Only 79 hospitals $(1.8 \%$ of all hospitals) caring for patients with acute myocardial infarction and 162 hospitals (3.4\% of hospitals) caring for patients with pneumonia had more than 25 index admissions and no readmissions during the study period. More than $93 \%$ of these "super performing" hospitals for acute myocardial infarction and $96 \%$ of these "super performing" hospitals for pneumonia did not submit survey data to the American Hospital Association and are therefore difficult to characterize. Study conclusions might therefore not apply to hospitals not participating in the annual survey of the American Hospital Association. This study of more than 600000 hospital readmissions from more than 4000 hospitals, however, is the largest of its kind and shows consistent findings across the most common cardiopulmonary conditions. Though we relied on claims data to assign diagnoses to admissions, administrative codes have been validated for cardiovascular and pulmonary diagnoses. ${ }^{51-53} \mathrm{We}$ did not exclude all potential planned readmissions such as those for treatment with chemotherapy or radiotherapy. However, we did not expect these elective admissions to be common soon after incident heart failure, myocardial infarction, or major infection such as pneumonia. We did exclude readmissions for revascularization after admission for acute myocardial infarction. Lastly, there might be concerns that the hierarchical modeling approach used by the Centers for Medicare and Medicaid Services obscures relations because findings are weighted by hospital volume, thereby making it less likely that small hospitals are considered high or low performers. This approach, however, was approved by the National Quality Forum and an independent committee of statisticians to reduce the likelihood of finding spurious results based on small sample volumes at certain hospitals. Moreover, we observed a substantial range of readmissions with this method. Our goal was not to further validate the readmission performance algorithms created by the Centers for Medicare and Medicaid Services but to understand how differences in hospitals' risk standardized readmission rates relate to readmission patterns.

\section{Conclusions}

We found that in contemporary practice, readmission diagnoses and timing after admission for heart failure, acute myocardial infarction, or pneumonia do not differ by hospital 30 day risk standardized readmission rates. Rather than having a smaller number of readmissions from particular diagnoses or time periods after discharge, high performing hospitals have fewer readmissions across the broad spectrum of readmission diagnoses throughout the post-discharge period. These findings suggest that lower readmission rates might best be achieved through use of general strategies and capacities that lower readmission risk globally rather than for specific diagnoses or time periods after admission.

Contributors: KD and HMK devised the study concept and design; analyzed and interpreted the data; drafted the paper; and are guarantors. AFH and ZL performed all statistical analyses. AFH, ZL, HB, JSR, LIH, JAB-F, NK, LGS, LMB, SMB, and EED analyzed and interpreted the data and critically revised the manuscript for important intellectual content. HMK obtained funding, acquired the data, and supervised the study. KD and HMK had full access to all the data in the study and take responsibility for the integrity of the data and the accuracy of the data analyses.

Funding: This study was funded by the National Heart, Lung, and Blood Institute, which had no role in the design, conduct, or analysis of the study or in the decision to submit the manuscript for publication. KD 


\section{What is already known on this topic}

Rates of readmission after hospital admission for heart failure, acute myocardial infarction, and pneumonia vary greatly across hospitals It is unknown whether high performing hospitals with low 30 day readmission rates have a lower proportion of readmissions from specific diagnoses and in certain time periods after admission or instead have a similar distribution of readmission diagnoses and timing as low performing institutions with high rates of readmission

\section{What this study adds}

The distribution of readmissions by diagnosis and the median time to readmission were similar across hospital performance categories for all three conditions studied

Rather than have a smaller number of readmissions from particular diagnoses or time periods after discharge, high performing hospitals have fewer readmissions across the broad spectrum of readmission diagnoses throughout the post-discharge period

Lower readmission rates might be best achieved through the use of general strategies and capacities that lower readmission risk globally rather than interventions that target specific diagnoses or time periods after admission

was supported by grant T32HL007854 from the National Heart, Lung, and Blood Institute and as a Centers of Excellence Scholar in Geriatric Medicine at Yale by the John A Hartford Foundation and the American Federation for Aging Research during the time that the work was conducted. JSR and LIH are supported by grants K08AG032886 and K08AG038336 from the National Institute on Aging and by the American Federation for Aging Research through the Paul B Beeson Career Development Award Program, respectively. JAB-F was supported by grant 3436-10-1 from CAPES (Coordenação de Aperfeicoamento de Pessoal de Nível Superior, Ministry of Education, Brazil) during the time that the work was conducted. HMK is supported by grant 1U01HL105270-03 (Center for Cardiovascular Outcomes Research at Yale University) from the National Heart, Lung, and Blood Institute. The content is solely the responsibility of the authors and does not necessarily represent the official views of the National Institutes of Health or the American Federation for Aging Research.

Competing interests: All authors have completed the ICMJE uniform disclosure form at www.icmje.org/coi_disclosure.pdf (available on request from the corresponding author) and declare: ZL, JSR, LIH, NK, LGS, SMB, EED, and HMK work under contract with the Centers for Medicare and Medicaid Services in the United States to develop and maintain performance measures; $\mathrm{HB}$ has received speaking or advisory fees from Astra-Zeneca, Bayer, Daiichi-Sankyo, Eli Lilly, Novartis, and Roche; JSR is a member of a scientific advisory board for FAIR Health; HMK is chair of a cardiac scientific advisory board for UnitedHealth; JSR and HMK are the recipients of a research grant from Medtronic through Yale University.

Ethical approval: The study received institutional review board approval through the Yale University Human Investigation.

Transparency declaration: KD affirms that this manuscript is an honest, accurate, and transparent account of the study being reported; that no important aspects of the study have been omitted; and that any discrepancies from the study as planned (and, if relevant, registered) have been explained.

Data sharing: No additional data available.

Krumholz HM, Merrill AR, Schone EM, Schreiner GC, Chen J, Bradley EH, et al. Patterns of hospital performance in acute myocardial infarction and heart failure 30-day mortality and readmission. Circ Cardiovasc Qual Outcomes 2009;2:407-13.

2 Bernheim SM, Grady JN, Lin Z, Wang Y, Savage SV, Bhat KR, et al. National patterns of risk-standardized mortality and readmission for acute myocardial infarction and heart failure. Update on publicly reported outcomes measures based on the 2010 release. Circ Cardiovasc Qual Outcomes 2010:3:459-67.

3 Lindenauer PK, Bernheim SM, Grady JN, Lin Z, Wang Y, Merrill AR, et al. The performance of US hospitals as reflected in risk-standardized 30-day mortality and readmission rates for Medicare beneficiaries with pneumonia. J Hosp Med 2010;5:E12-8.

4 Centers for Medicare and Medicaid Services. Medicare hospital quality chartbook 2011 performance report on readmission measures for acute myocardial infarction, heart failure, and pneumonia. Centers for Medicare and Medicaid Services, September 2011.

5 Centers for Medicare and Medicaid Services. Medicare hospital compare quality of care. www.hospitalcompare.hhs.gov/?AspxAutoDetectCookieSupport=1

6 Curry LA, Spatz E, Cherlin E, Thompson JW, Berg D, Ting HH, et al. What distinguishes top-performing hospitals in acute myocardial infarction mortality rates? A qualitative study. Ann Intern Med 2011:154:384-90.

7 Bradley EH, Curry LA, Spatz ES, Herrin J, Cherlin EJ, Curtis JP, et al. Hospital strategies for reducing risk-standardized mortality rates in acute myocardial infarction. Ann Intern Med 2012;156:618-26.
8 Agency for Healthcare Research and Quality. Statistical brief \#66. Healthcare Cost and Utilization Project (HCUP). Agency for Healthcare Research and Quality, 2009. www. hcup-us.ahrq.gov/reports/statbriefs/sb66.jsp.

9 Kocher RP, Adashi EY. Hospital readmissions and the Affordable Care Act: paying for coordinated quality care. JAMA 2011;306:1794-5.

10 Keenan PS, Normand SL, Lin Z, Drye EE, Bhat KR, Ross JS, et al. An administrative claims measure suitable for profiling hospital performance on the basis of 30-day all-cause readmission rates among patients with heart failure. Circ Cardiovasc Qual Outcomes 2008:1:29-37.

11 Lindenauer PK, Normand SL, Drye EE, Lin Z, Goodrich K, Desai MM, et al. Development, validation, and results of a measure of 30-day readmission following hospitalization for pneumonia. J Hosp Med 2011;6:142-50

12 Krumholz HM, Lin Z, Drye EE, Desai MM, Han LF, Rapp MT, et al. An administrative claims measure suitable for profiling hospital performance based on 30-day all-cause readmission rates among patients with acute myocardial infarction. Circ Cardiovasc Qual Outcomes 2011:4:243-52.

13 Pope GC, Kautter J, Ellis RP, Ash AS, Ayanian JZ, Lezzoni LI, et al. Risk adjustment of Medicare capitation payments using the CMS-HCC model. Health Care Financ Rev 2004;25:119-41.

14 Dharmarajan K, Hsieh AF, Lin Z, Bueno H, Ross JS, Horwitz LI, et al. Diagnoses and timing of 30-day readmissions after hospitalization for heart failure, acute myocardial infarction, or pneumonia. JAMA 2013;309:355-63.

15 National Quality Forum. National voluntary consensus standards for hospital care 2007 performance measures-a consensus report. National Quality Forum, 2008.

16 Ash AS, Fienberg SE, Louis TA, Normand S-LT, Stukel TA, Utts J. COPSS-CMS White Paper Committee. Statistical issues in assessing hospital performance. 2012. www.cms. gov/Medicare/Quality-Initiatives-Patient-Assessment-Instruments/HospitalQualitylnits/ Downloads/Statistical-Issues-in-Assessing-Hospital-Performance.pdf.

17 Joynt KE, Jha AK. Who has higher readmission rates for heart failure, and why? Implications for efforts to improve care using financial incentives. Circ Cardiovasc Qual Outcomes 2011;4:53-9.

18 Joynt KE, Orav EJ, Jha AK. The association between hospital volume and processes, outcomes, and costs of care for congestive heart failure. Ann Intern Med 2011:154:94-102.

19 Epstein AM, Jha AK, Orav EJ. The relationship between hospital admission rates and rehospitalizations. N Engl J Med 2011;365:2287-95.

20 Ross JS, Bernheim SM, Lin Z, Drye EE, Chen J, Normand SL, et al. Based on key measures, care quality for Medicare enrollees at safety-net and non-safety-net hospitals was almost equal. Health Aff 2012;31:1739-48.

21 Gaskin DJ, Hadley J, Freeman VG. Are urban safety-net hospitals losing low-risk Medicaid maternity patients? Health Serv Res 2001;36:25-51.

22 Jencks SF, Williams MV, Coleman EA. Rehospitalizations among patients in the Medicare fee-for-service program. N Engl J Med 2009;360:1418-28.

23 O'Connor CM, Miller AB, Blair JE, Konstam MA, Wedge P, Bahit MC, et al. Causes of death and rehospitalization in patients hospitalized with worsening heart failure and reduced left ventricular ejection fraction: results from Efficacy of Vasopressin Antagonism in Heart Failure Outcome Study with Tolvaptan (EVEREST) program. Am Heart $J$ 2010;159:841-49.

24 Jasti H, Mortensen EM, Obrosky DS, Kapoor WN, Fine MJ. Causes and risk factors for rehospitalization of patients hospitalized with community-acquired pneumonia. Clin Infect Dis 2008:46:550-6.

25 Dunlay SM, Weston SA, Killian JM, Bell MR, Jaffe AS, Roger VL. Thirty-day rehospitalizations after acute myocardial infarction: a cohort study. Ann Intern Med 2012;157:11-8.

26 Joynt KE, Orav EJ, Jha AK. Thirty-day readmission rates for Medicare beneficiaries by race and site of care. JAMA 2011;305:675-81.

27 Hansen LO, Young RS, Hinami K, Leung A, Williams MV. Interventions to reduce 30-day rehospitalization: a systematic review. Ann Intern Med 2011;155:520-8.

28 Rich MW, Beckham V, Wittenberg C, Leven CL, Freedland KE, Carney RM. A multidisciplinary intervention to prevent the readmission of elderly patients with congestive heart failure. N Engl J Med 1995;333:1190-5.

29 Coleman EA, Parry C, Chalmers S, Min SJ. The care transitions intervention: results of a randomized controlled trial. Arch Intern Med 2006;166:1822-8.

30 Chaudhry SI, Mattera JA, Curtis JP, Spertus JA, Herrin J, Lin Z, et al. Telemonitoring in patients with heart failure. N Engl J Med 2010;363:2301-9.

31 Dudas V, Bookwalter T, Kerr KM, Pantilat SZ. The impact of follow-up telephone calls to patients after hospitalization. Am J Med 2001;111:26S-30S.

32 Koehler F, Winkler S, Schieber M, Sechtem U, Stangl K, Bohm M, et al. Impact of remote telemedical management on mortality and hospitalizations in ambulatory patients with chronic heart failure: the telemedical interventional monitoring in heart failure study. Circulation 2011;123:1873-80.

33 Joynt KE, Harris Y, Orav EJ, Jha AK. Quality of care and patient outcomes in critical access rural hospitals. JAMA 2011;306:45-52.

34 Baldwin LM, Chan L, Andrilla CH, Huff ED, Hart LG. Quality of care for myocardial infarction in rural and urban hospitals. J Rural Health 2010;26:51-7. 
35 Sheikh K, Bullock C. Urban-rural differences in the quality of care for Medicare patients with acute myocardial infarction. Arch Intern Med 2001:161:737-43.

36 Nawal Lutfiyya M, Bhat DK, Gandhi SR, Nguyen C, Weidenbacher-Hoper VL, Lipsky MS. A comparison of quality of care indicators in urban acute care hospitals and rural critical access hospitals in the United States. Int J Qual Health Care 2007;19:141-9.

37 Hartman L, Jarosek SL, Virnig BA, Durham S. Medicare-certified home health care: urban-rural differences in utilization. J Rural Health 2007;23:254-7.

38 Chan L, Hart LG, Goodman DC. Geographic access to health care for rural Medicare beneficiaries. J Rural Health 2006;22:140-6.

39 Probst JC, Laditka SB, Wang JY, Johnson AO. Effects of residence and race on burden of travel for care: cross sectional analysis of the 2001 US National Household Travel Survey. BMC Health Serv Res 2007;7:40.

40 Calvillo-King L, Arnold D, Eubank KJ, Lo M, Yunyongying P, Stieglitz H, et al. Impact of social factors on risk of readmission or mortality in pneumonia and heart failure: systematic review. J Gen Intern Med 2013;28:269-82.

41 Shah SJ, Krumholz HM, Reid KJ, Rathore SS, Mandawat A, Spertus JA, et al. Financial stress and outcomes after acute myocardial infarction. PLOS One 2012;7:e47420.

42 Bernheim SM, Spertus JA, Reid KJ, Bradley EH, Desai RA, Peterson ED, et al. Socioeconomic disparities in outcomes after acute myocardial infarction. Am Heart $J$ 2007;153:313-9.

43 Bucholz EM, Rathore SS, Gosch K, Schoenfeld A, Jones PG, Buchanan DM, et al. Effect of living alone on patient outcomes after hospitalization for acute myocardial infarction. Am J Cardiol 2011;108:943-8

44 Howie-Esquivel J, Spicer JG. Association of partner status and disposition with rehospitalization in heart failure patients. Am J Crit Care 2012;21:e65-73.

45 Evangelista LS, Doering LV, Dracup K. Usefulness of a history of tobacco and alcoho use in predicting multiple heart failure readmissions among veterans. Am J Cardiol 2000;86:1339-42.

46 Neupane B, Walter SD, Krueger P, Marrie T, Loeb M. Predictors of inhospital mortality and re-hospitalization in older adults with community-acquired pneumonia: a prospective cohort study. BMC Geriatr 2010;10:22.
47 Hammill BG, Curtis LH, Fonarow GC, Heidenreich PA, Yancy CW, Peterson ED, et al. Incremental value of clinical data beyond claims data in predicting 30-day outcomes after heart failure hospitalization. Circ Cardiovasc Qual Outcomes 2011;4:60-7.

48 Kaboli PJ, Go JT, Hockenberry J, Glasgow JM, Johnson SR, Rosenthal GE, et al. Associations between reduced hospital length of stay and 30-day readmission rate and mortality: 14-year experience in 129 Veterans Affairs hospitals. Ann Intern Med 2012;157:837-45.

49 Department of Health and Human Services. Progress report from CMS before the US Senate Finance Committee. February 28, 2013. www.finance.senate.gov/imo/media/doc/ CMS\%20Delivery\%20System\%20Reform\%20Testimony\%202.28.13\%20(J.\%20Blum). pdf.

50 Baldrige Performance Excellence Program. 2013-2014 Health Care Criteria for Performance Excellence. 2013. www.nist.gov/baldrige/publications/hc_criteria.cfm.

51 Birman-Deych E, Waterman AD, Yan Y, Nilasena DS, Radford MJ, Gage BF. Accuracy of ICD-9-CM codes for identifying cardiovascular and stroke risk factors. Med Care 2005;43:480-5.

52 Kiyota Y, Schneeweiss S, Glynn RJ, Cannuscio CC, Avorn J, Solomon DH. Accuracy of Medicare claims-based diagnosis of acute myocardial infarction: estimating positive predictive value on the basis of review of hospital records. Am Heart J 2004;148:99-104.

53 Skull SA, Andrews RM, Byrnes GB, Campbell DA, Nolan TM, Brown GV, et al. ICD-10 codes are a valid tool for identification of pneumonia in hospitalized patients aged $>$ or $=$ 65 years. Epidemiol Infect 2008;136:232-40.

\section{Accepted: 21 October 2013}

\section{Cite this as: BMJ 2013;347:f6571}

This is an Open Access article distributed in accordance with the Creative Commons Attribution Non Commercial (CC BY-NC 3.0) license, which permits others to distribute, remix, adapt, build upon this work non-commercially, and license their derivative works on different terms, provided the original work is properly cited and the use is non-commercial. See: http://creativecommons.org/licenses/by-nc/3.0/. 


\section{Tables}

Table 1| Hospital characteristics by reason for index admission and performance category for readmissions (high performing hospitals have low rates of readmission). All numbers are percentages unless stated otherwise

\begin{tabular}{|c|c|c|c|c|}
\hline & \multicolumn{4}{|c|}{ Hospital performance categories } \\
\hline & All & High performing & Average performing & Low performing \\
\hline \multicolumn{5}{|l|}{ Heart failure } \\
\hline No of hospitals & 4041 & 133 & 3692 & 216 \\
\hline \multicolumn{5}{|l|}{ Median (IQR): } \\
\hline 30 day risk standardized readmission rate ${ }^{*}$ & $24.7(23.6-26.0)$ & $21.1(20.3-21.6)$ & $24.6(23.6-25.8)$ & $28.9(27.9-29.5)$ \\
\hline Annual heart failure volume & $64.0(27.7-146.3)$ & $202.0(158.3-311.3)$ & $57.0(25.5-130.0)$ & $170.5(99.5-293.5)$ \\
\hline Medicaid $†$ & $15.8(10.5-20.6)$ & $16.1(11.2-19.6)$ & $15.7(10.3-20.3)$ & $18.8(13.4-24.9)$ \\
\hline African-American $\ddagger$ & $2.8(0.0-12.2)$ & $3.2(0.7-9.6)$ & $2.5(0.0-11.2)$ & $11.7(2.7-32.9)$ \\
\hline Rural hospital & 22.4 & 1.5 & 23.7 & 10.6 \\
\hline Critical access hospital & 23.1 & 1.5 & 25.1 & 1.9 \\
\hline Teaching hospital & 18.7 & 39.8 & 16.8 & 37.5 \\
\hline Safety net hospital§ & 29.8 & 18.0 & 30.5 & 25.0 \\
\hline Publicly owned hospital & 22.1 & 12.0 & 22.9 & 13.4 \\
\hline Not for profit hospital & 62.1 & 78.9 & 61.1 & 68.1 \\
\hline Privately owned hospital & 15.9 & 9.0 & 16.0 & 18.5 \\
\hline \multicolumn{5}{|l|}{ AMI } \\
\hline No of hospitals & 2378 & 73 & 2225 & 80 \\
\hline \multicolumn{5}{|l|}{ Median (IQR): } \\
\hline 30 day risk standardized readmission rate ${ }^{*}$ & $19.8(18.7-21.1)$ & $16.1(15.7-16.6)$ & $19.8(18.8-21.0)$ & $24.6(23.7-25.6)$ \\
\hline Annual acute myocardial infarction volume & $44.3(19.3-96.0)$ & $152.3(110.7-256.0)$ & $40.0(18.3-87.3)$ & $127.5(64.3-213.0)$ \\
\hline Medicaid $†$ & $17.5(12.2-21.7)$ & $14.9(11.3-19.1)$ & $17.5(12.1-21.7)$ & $19.5(16.3-29.4)$ \\
\hline African-American $\ddagger$ & $3.1(0.5-9.7)$ & $1.4(0.4-3.5)$ & $3.0(0.5-9.2)$ & $14.2(4.5-30.6)$ \\
\hline Rural hospital & 5.1 & 0.0 & 5.3 & 2.5 \\
\hline Critical access hospital & 2.0 & 0.0 & 2.1 & 0.0 \\
\hline Teaching hospital & 29.1 & 39.7 & 27.3 & 31.3 \\
\hline Safety net hospital§ & 22.0 & 12.3 & 22.2 & 26.3 \\
\hline Publicly owned hospital & 13.0 & 8.2 & 13.3 & 8.8 \\
\hline Not for profit hospital & 69.4 & 80.8 & 68.6 & 82.5 \\
\hline Privately owned hospital & 17.6 & 11.0 & 18.2 & 8.8 \\
\hline \multicolumn{5}{|l|}{ Pneumonia } \\
\hline No of hospitals & 4283 & 71 & 4040 & 172 \\
\hline \multicolumn{5}{|l|}{ Median (IQR): } \\
\hline 30 day risk standardized readmission rate ${ }^{*}$ & $18.2(17.3-19.1)$ & $15.1(14.6-15.5)$ & $18.2(17.3-19.2)$ & $22.3(22.1-23.0)$ \\
\hline Annual pneumonia volume & $65.3(33.0-119.3)$ & $157.0(110.3-209.0)$ & $61.3(31.7-112.7)$ & $140.0(95.8-202.5)$ \\
\hline Medicaidt & $15.6(10.1-20.2)$ & $15.7(11.8-19.5)$ & $15.5(10.0-20.2)$ & $17.6(12.3-21.8)$ \\
\hline African-American $\ddagger$ & $1.6(0.0-7.5)$ & $1.1(0.2-3.9)$ & $1.5(0.0-7.2)$ & $6.9(1.3-16.0)$ \\
\hline Rural hospital & 25.3 & 4.2 & 26.1 & 15.1 \\
\hline Critical access hospital & 26.8 & 7.0 & 28.1 & 3.5 \\
\hline Teaching hospital & 17.8 & 38.0 & 16.6 & 36.0 \\
\hline Safety net hospital§ & 31.4 & 30.9 & 31.8 & 21.5 \\
\hline Publicly owned hospital & 24.0 & 21.1 & 24.4 & 14.5 \\
\hline Not for profit hospital & 61.0 & 73.2 & 60.4 & 70.3 \\
\hline Privately owned hospital & 15.1 & 5.6 & 15.2 & 15.1 \\
\hline
\end{tabular}

IQR=interquartile range. 
Table 1 (continued)

Hospital performance categories

All High performing Average performing Low performing

*Over three years.

†Median hospital proportion of patients with Medicaid health insurance.

$\ddagger$ Median hospital proportion of African-American patients.

$\S$ Safety net hospitals in US provide care for high proportion of uninsured, underinsured, or Medicaid patients; we have defined safety net hospitals as public or private hospitals with annual Medicaid caseload $>1$ SD above their respective state's mean private hospital Medicaid caseload. 
Table 2| Relation between hospital characteristics and median time to readmission after index admission to hospital for heart failure, acute myocardial infarction, or pneumonia

\begin{tabular}{|c|c|c|c|}
\hline \multirow[b]{2}{*}{ Hospital characteristic } & \multicolumn{3}{|c|}{ Coefficient for change in median time to readmission in days $(95 \% \mathrm{Cl})$} \\
\hline & Heart failure & Acute myocardial infarction & Pneumonia \\
\hline $\begin{array}{l}30 \text { day risk standardized readmission } \\
\text { rate }^{*}\end{array}$ & $-0.15(-0.19$ to -0.10$)$ & $0.03(-0.05$ to 0.11$)$ & $-0.23(-0.29$ to -0.17$)$ \\
\hline Annual hospital volume $\dagger$ & $0.01(-0.03$ to 0.05$)$ & $-0.11(-0.17$ to -0.05$)$ & 0.07 (0.02 to 0.12$)$ \\
\hline$\%$ Medicaid $\neq$ & $-0.06(-0.12$ to 0.01$)$ & $-0.01(-0.13$ to 0.10$)$ & $-0.07(-0.16$ to 0.01$)$ \\
\hline$\%$ African-American§ & $0.11(0.06$ to 0.17$)$ & $0.04(-0.07$ to 0.14$)$ & $0.03(-0.04$ to 0.10$)$ \\
\hline Rural hospitalq & $0.10(-0.14$ to 0.34$)$ & $-0.25(-1.00$ to 0.50$)$ & $0.24(-0.00$ to 0.48$)$ \\
\hline Critical access hospital & $-0.07(-0.36$ to 0.21$)$ & $0.45(-1.02$ to 1.92$)$ & $0.29(0.01$ to 0.56$)$ \\
\hline 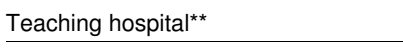 & $-0.09(-0.21$ to 0.03$)$ & $-0.22(-0.40$ to -0.03$)$ & $-0.15(-0.30$ to -0.01$)$ \\
\hline Safety net hospitalt† & $0.07(-0.15$ to 0.29$)$ & $0.05(-0.32$ to 0.42$)$ & $0.22(-0.06$ to 0.49$)$ \\
\hline Publicly owned hospitalł‡ & $-0.05(-0.32$ to 0.22$)$ & $-0.08(-0.55$ to 0.40$)$ & $-0.32(-0.66$ to 0.02$)$ \\
\hline Not for profit hospital§§ & $-0.02(-0.36$ to 0.21$)$ & $0.19(-0.08$ to 0.45$)$ & $-0.13(-0.32$ to 0.05$)$ \\
\hline
\end{tabular}

*Over three years. Coefficient for change for every SD increase in rate. For example, for heart failure readmission cohort, when rate increased by 1 SD, hospital median time to readmission decreased by 0.148 days.

†Total No of admissions for index condition 2007-09 divided by 3. Coefficient for change for every increase in annual volume for index condition by 1 SD.

¥Proportion of patients with Medicaid health insurance cared for by facility as defined with 2008 American Hospital Association data. Coefficient for change for every $1 \mathrm{SD}$ increase in proportion of hospital patients that have Medicaid health insurance.

§Proportion of African-American patients cared for by facility in 2007-09. Coefficient for change every 1 SD increase in proportion of African-American hospital patients.

TRural $v$ urban.

**Teaching $v$ non-teaching hospitals

††Safety net hospitals in US provide care for high proportion of uninsured, underinsured, or Medicaid patients; we have defined safety net hospitals as public or private hospitals with annual Medicaid caseload >1 SD above their respective state's mean private hospital Medicaid caseload.

¥†Publicly $v$ privately owned hospitals.

$\S \S$ Not for profit $v$ privately owned hospitals. 


\section{Figures}

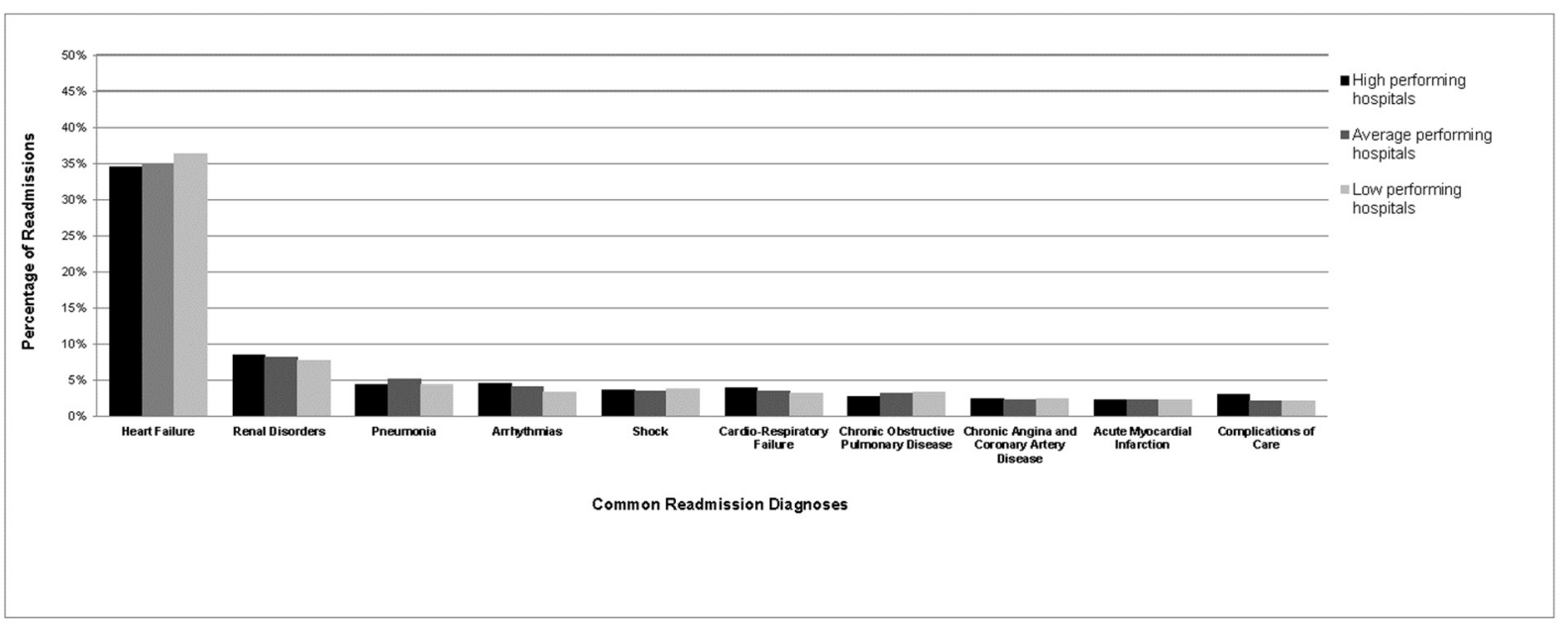

Fig 1 Ten most common readmission diagnoses for high, average, and low performing hospitals after admission for heart failure. Numbers rounded to nearest percentage point. High performing hospitals have 30 day risk standardized readmission rates lower than national average rate with $>95 \%$ probability. Low performing hospitals have 30 day risk standardized readmission rates higher than national average rate with $>95 \%$ probability. All remaining hospitals are considered average. For each hospital, we calculated performance separately for heart failure, acute myocardial infarction, and pneumonia cohorts

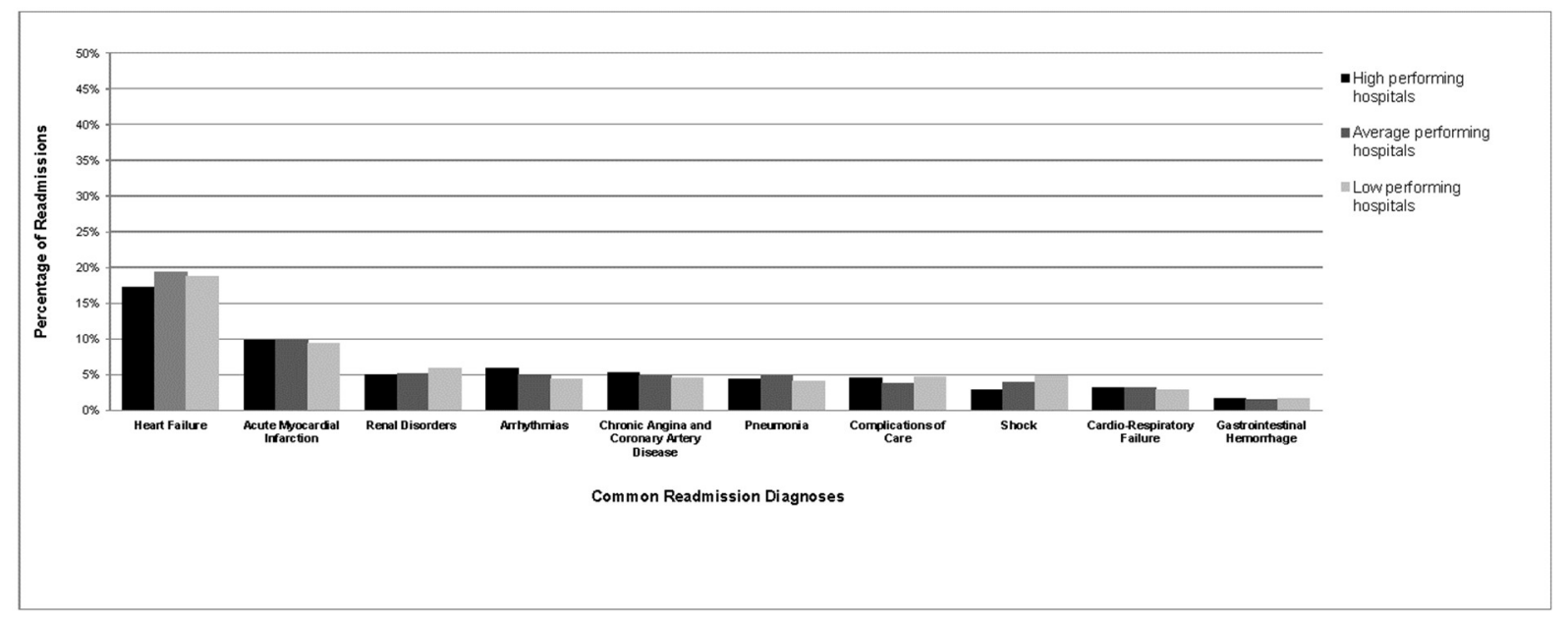

Fig 2 Ten most common readmission diagnoses for high, average, and low performing hospitals after admissions for acute myocardial infarction. See also legend for fig 1 


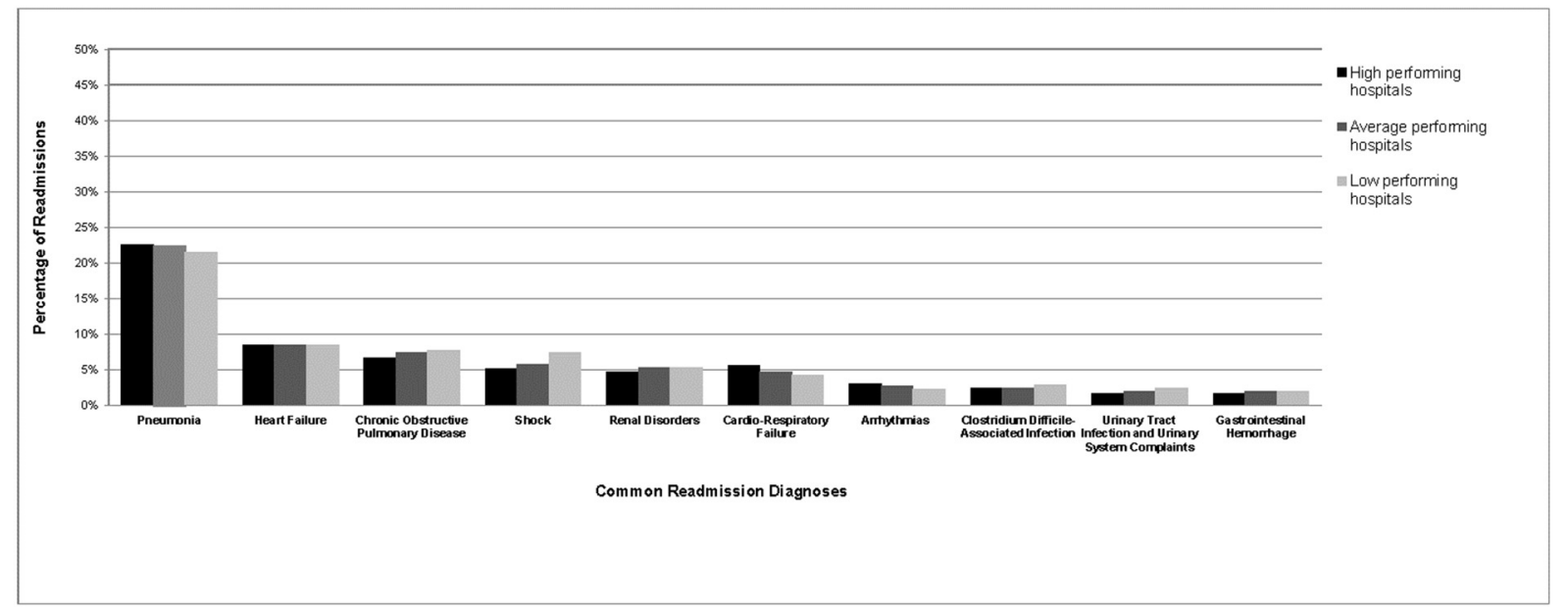

Fig 3 Ten most common readmission diagnoses for high, average, and low performing hospitals after admission for pneumonia. See also legend for fig 1

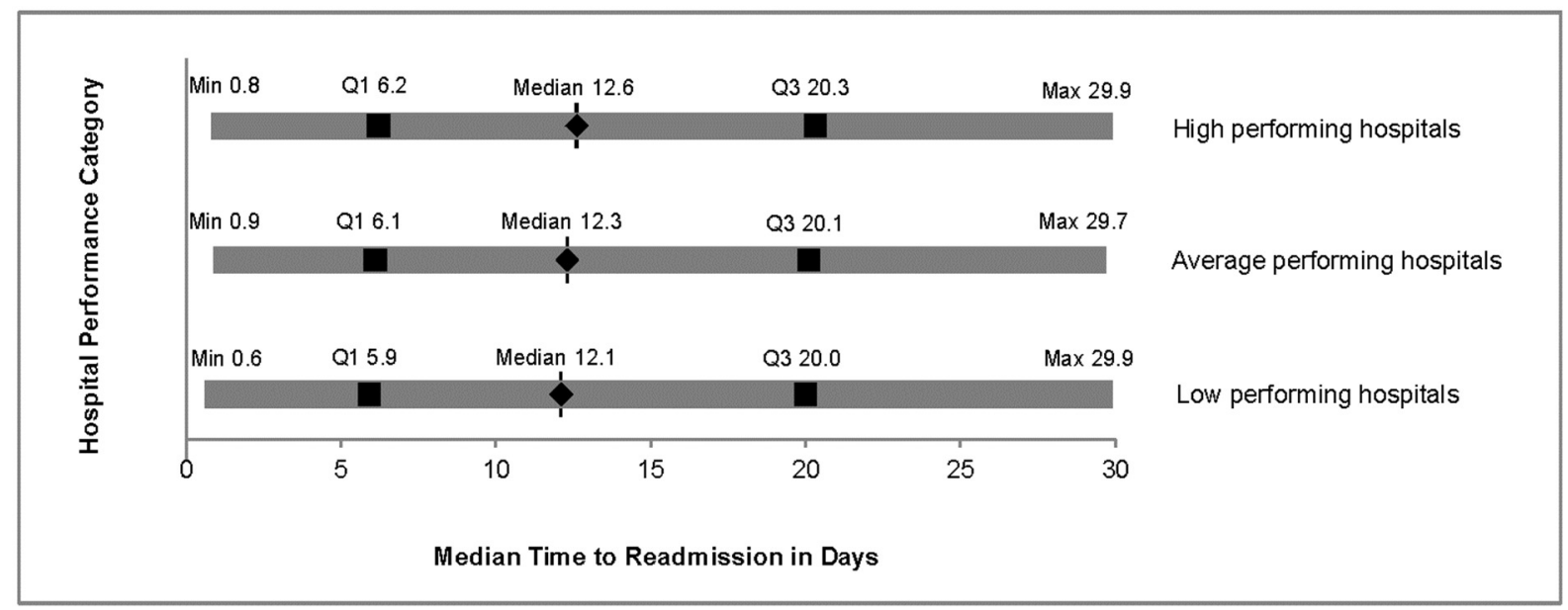

Fig 4 Weighted median time to readmission for high, average, and low performing hospitals after admission for heart failure. High performing hospitals have 30 day risk standardized readmission rates lower than national rate with $>95 \%$ probability. Low performing hospitals have 30 day risk standardized readmission rates higher than national average rate with $>95 \%$ probability. All remaining hospitals are considered average. For each hospital, we calculated performance separately for heart failure, acute myocardial infarction, and pneumonia cohorts. Weighted median time to readmission denoted by black diamond; interquartile range denoted by black squares; range denoted by gray bar 


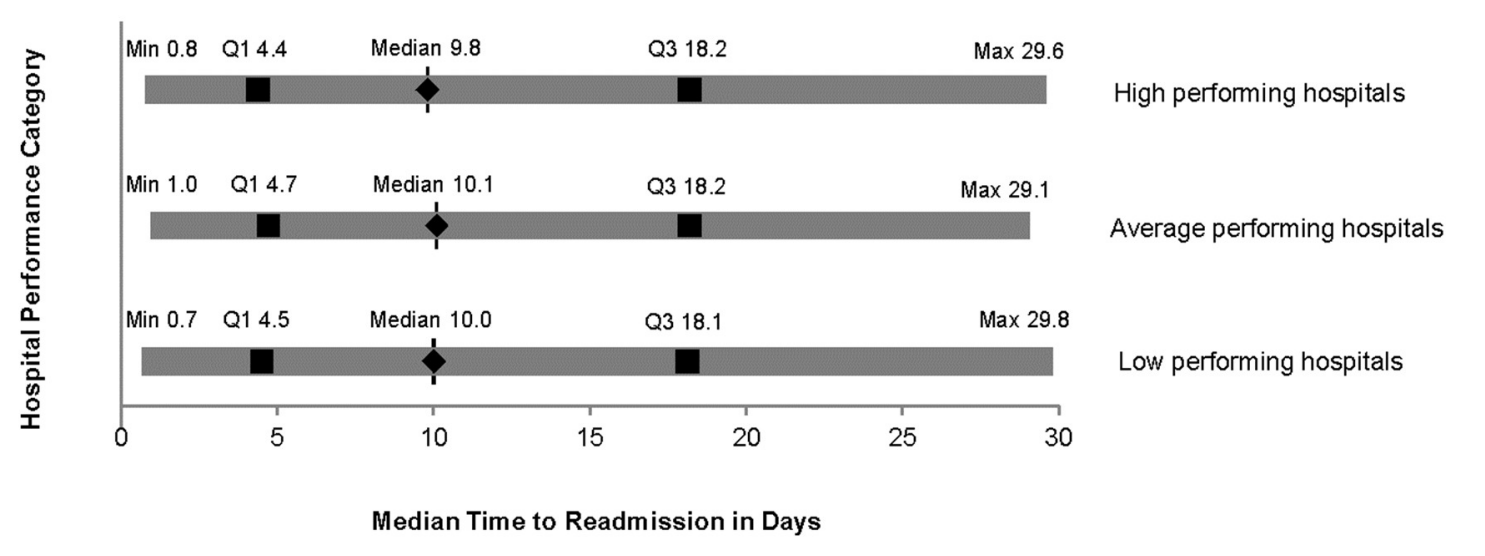

Fig 5 Weighted median time to readmission for high, average, and low performing hospitals after admission for acute myocardial infarction. See also legend for fig 4

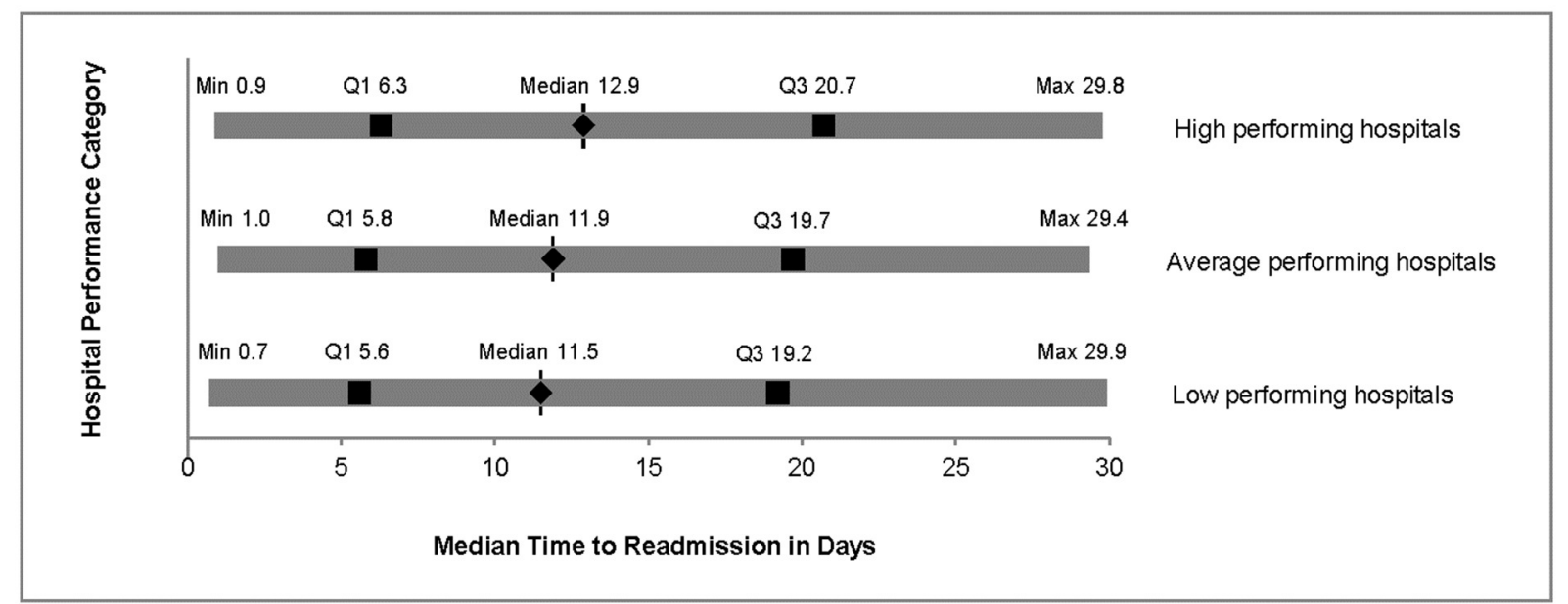

Fig 6 Weighted median time to readmission for high, average, and low performing hospitals after admission for pneumonia. See also legend for fig 4 